\title{
Three Histidines in Pterin-4a-carbinolamine Dehydratase are the Important Residues for Substrate Binding and Catalysis
}

\author{
Sandra Köster ${ }^{1}$, Gunter Stier ${ }^{2}$, Ralf Ficner ${ }^{2}$, Hans-Christoph Curtius ${ }^{1}$, Dietrich Suck ${ }^{2}$ and Sandro Ghisla ${ }^{1 \S}$ \\ ${ }^{1}$ Department of Biology, University of Konstanz, P.O. Box 5560, D-78434 Konstanz, Germany \\ ${ }^{2}$ EMBL, Structural Biology Programme, Meyerhofstr. 1, D-69117 Heidelberg, Germany
}

\section{Introduction}

Pterin-4a-carbinolamine dehydratase/dimerization cofactor of hepatocyte nuclear factor- $1 \alpha$ $(\mathrm{PCD} / \mathrm{DCoH})$ is a bifunctional protein. Its catalytic function consists in the dehydration of pterin-4a-carbinolamine to quinonoid 7,8-dihydrobiopterin ( 1 ); the substrate, pterin-4a-carbinolamine, is the product arising from the pterin cofactor during the aromatic hydroxylation catalyzed by pterin dependent hydroxylases. The second function consists in the activation, via induction of dimerization, of the transcription factor hepatocyte nuclear factor-l $\alpha$ (2). The recombinant form of hwt-PCD has been purified and characterized $(3,4)$ and the three-dimensional structure has been solved $(5,6)$. The aim of our studies was the identification of the active site of $\mathrm{PCD} / \mathrm{DCoH}$ and the elucidation of the catalytic mechanism. Using site-directed mutagenesis we have studied the involvement of residues which, based on the $3 \mathrm{D}$-structure $(5,6)$ (see also Fig. 1), might be at the presumed active site and might be involved in catalysis. Details of the data presented here have been submitted to EJB for publication (7).

\section{Materials and Methods}

The recombinant protein contains a $\mathrm{N}$-terminal histidine tag and was purified by affinity chromatography over a $\mathrm{Ni}^{2+}$-nitriloacetic acid-agarose

§ Author to whom correspondence should be addressed. column as detailed in (7). Subsequently the Histag was cleaved with thrombin (7). Dehydratase activity was measured in a so called direct assay using 6-methyl-4a-carbinolamine as substrate (prepared according to Bailey et al. (8) with slight modifications (3)). In a sample cuvette containing PCD (from 0.1 to $1 \mu \mathrm{M}$ ) substrate was added (up to $140 \mu \mathrm{M}$ ) and the dehydration followed spectrophotometrically at $\lambda=245 \mathrm{~nm}$. Conditions of the assays are given in the legend of the Table. The determination of binding constants based on the quenching of the tryptophane fluorescence of PCD induced by ligand binding was achieved as described earlier (3).

\section{Results}

The results obtained from the study of point mutations in PCD are summarized in the Table. In addition to the residues exchanged earlier (7) also the Y69A- and H62A,H79A-PCD mutants have been expressed and purified. H61A-PCD and H62A-PCD have $\approx 10 \%$ the activity of hwtPCD, however, mutation of both histidines to form the double mutant H61A,H62A-PCD leads to a complete loss of activity. The $\mathrm{K}_{\mathrm{m}}$ obtained using 6-methyl-4a-carbinolamine as substrate in the direct assay is about tenfold higher for H6lAPCD compared to that for hwt-PCD. H62APCD is the only mutant which shows a significant shift of the $\mathrm{pK}$ of the observed dehydration rate (Table), again compared to hwt-PCD. From the 3D-representation (cf. Fig. 1) it can be deduced that His61 and His62 are in hydrogen bonding 
contact with Asp88 and Glu57 (Fig. 1), respectively. The D88N-PCD forms inclusion bodies, whereas the E57A-PCD is the only single mutant devoid of activity. Most probably mutation of Glu 57 leads to structural rearrangements at the active center, consistent also with the low affinity for the

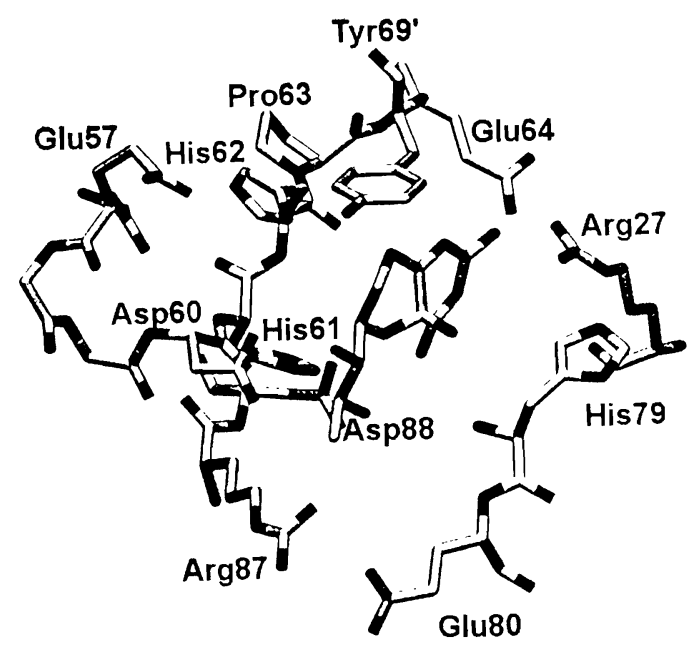

Figure 1. Active site residues of hwt-PCD with a docked $4 \mathrm{a}(\mathrm{S})-\mathrm{OH}-7,8$-dihydrobiopterin molecule. The docking was achieved using the program SETOR [10]. His61 and His62 are in hydrogen bonding distance to either $\mathrm{N}(5)-\mathrm{H}$ or $\mathrm{N}(8)-\mathrm{H}$ of the substrate and are able to act as deprotonating bases. They are in hydrogen bond contact with Asp88 and Glu57, respectively. His79 or a water molecule can attack the substrate from the opposite face and might protonate the $4 \mathrm{a}-\mathrm{OH}$ group acting as a general acid (adapted from [7]). quinonoid 6,6-dimethyl-7,8-dihydropterin.

The activity of H79A-PCD is reduced to $\approx 25 \%$ compared to that of hwt-PCD and the second double mutant H62A,H79A-PCD also shows no activity. Interestingly, the $\mathrm{Kd}$ for binding of quinonoid 6,6-dimethyl-7,8-dihydropterin to this double mutant is similar to that what was found for H62A-PCD. A further residue of interest is Tyr69. It belongs to the neighboring subunit and lies directly in front of the assumed binding pocket (Fig. 1). Exchange of Tyr69 leads to $\approx 40 \%$ activity in the case of Y69F-, Y69A- and Y69TPCD. The $\mathrm{K}_{\mathrm{m}}$ 's for these mutants are lower compared to that for hwt-PCD (Table) suggesting a less restricted access to the binding pocket. The

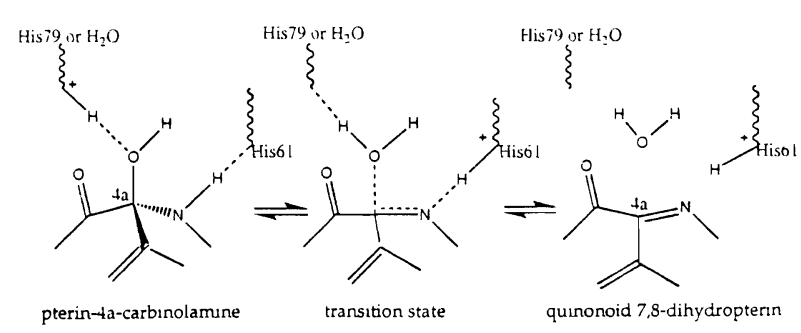

Scheme 1. Proposed catalytic mechanism of the dehydration of pterin-4a-carbinolamine to quinonoid 7, 8-dihydropterin. His61 is in hydrogen bonding distance to $\mathrm{N}(5)$ $\mathrm{H}$ and is able to act as general base catalyst. His79 or a water molecule can act as general acid catalysts by attack on the $4 \mathrm{a}-\mathrm{OH}$ group. Alternatively, His 62 could attack the $\mathrm{N}(8)-\mathrm{H}$ as dicussed in the text (not shown in the Scheme).

Table 1. Catalytic prooperties of hwt-PCD and selected mutants and binding of quinonoid 6,6-dimethyl-7,8-dihydropterin to PCD. Kinetic constants were calculated from the dehydration rates of 6-methyl-pterin-4a-carbinolamine followed at $245 \mathrm{~nm}$ in $10 \mathrm{mM}$ Tris-HCI pH 8.5 at $10^{\circ} \mathrm{C}$. Enzyme catalyzed assays have been corrected for the spontaneous decay. $\mathrm{pK}$ values were estimated from plots of the activity at varying $\mathrm{pH}$ and in the presence of a constant electrolyte concentration of $0.1 \mathrm{M} \mathrm{KCl} . \mathrm{V}_{\max }$ is expressed as the turnover number, i. e., nmol of substrate dehydrated per nmol of enzyme subunit per seczond (adapted in part from [7]).

\begin{tabular}{lccccc}
\hline \hline \multicolumn{1}{c}{ Enzyme } & $\mathrm{K}_{\mathrm{m}}(\mu \mathrm{M})$ & $\mathrm{V}_{\max }(\mathrm{nmol} / \mathrm{sec})$ & $(\%)$ & $\mathrm{pK}$ & $\mathrm{Kd}$ for binding of product $(\mu \mathrm{M}) \mathrm{a})$ \\
\hline hwt-PCD & 25 & 8.6 & 100 & 8.2 & 0.9 \\
E57A & & 0 & 0 & & $>165$ \\
H61A & 220 & 1 & 12 & 8.0 & $>55$ \\
H62A & 75 & 0.9 & 10 & 7.15 & $>65$ \\
H79A & 10 & 2.2 & 25 & 7.8 & 5 \\
H79S & 12 & 3.6 & 40 & n.d. & 2 \\
H61A, H62A & & 0 & 0 & & $>140$ \\
H62A, H79A & & 0 & 0 & & 4.4 \\
Y69F & 8 & 3.5 & 40 & 8.3 & 10 \\
Y69A & 10 & 3.9 & 45 & 8.2 & 3.8 \\
Y69T & 7 & 1.7 & 20 & 8.7 & \\
\hline
\end{tabular}

a) Upon excitation at $\lambda=280 \mathrm{~nm}$ PCD has an emission $\lambda_{\max } \approx 334 \mathrm{~nm}$. Addition of excess quinonoid 6,6-dimethyl-7,8dihydropterin to $1.1 \mu \mathrm{M}$ PCD in $0.1 \mathrm{M}$ Tris-HCI pH 8.4 leads to nearly complete quenching of the protein fluorescence emission. Kd's were determined by plotting the pterin concentration against the relative fluorescence, which leads to a monophasic saturation curve. 
behavior of these mutants is also different from hwt-PCD when activity is monitored at $\lambda=245$ $\mathrm{nm}$ and at $\mathrm{pH} \geq 8.5$. Thus, while the course of the reaction is monophasic with hwt-PCD and all other mutants, the behavior of the Tyr69 mutants cannot be fitted to a single or biphasic (two-exponential) process. This suggests that specific interactions exist, which could affect either binding or catalysis.

\section{Conclusions}

The catalytic properties of the mutants listed in Table 1 are compatible with the active site being located in the vicinity of His61, His62 and His79 as shown in Fig. 1 (7). The properties of the insoluble D88N mutant and of the inactive E57A mutant suggest that Asp88 and Glu57 might be responsible for keeping the two histidines 61 and 62 in the correct orientation and ionization. The two pairs are in H-bonding contact. His6l and His62 are also in appropriate distance and orientation to act as base catalysts by attacking either $\mathrm{N}(5)-\mathrm{H}$ or $\mathrm{N}(8)-\mathrm{H}$ of the pterin-4a-carbinolamine substrate. The residual activity of $\approx$ $10 \%$ observed with both single mutants is compatible with one histidine being able to exert the function of the other and vice versa. A peculiarity of PCD is its equal effectiveness in catalyzing the dehydration of the two possible substrate conformers at pos. 4a-OH. This is compatible with the assumption that His79 or His62 (or a water molecule linked to these residues) can act as general acid/base catalysts from either side of the active center to induce dehydration. Based on the results of Sayer et al. (9), which indicate general acid/base catalysis as the likely mechanism for the dehydration of this substrate type, we think that a mechanism for dehydration of pterin-4a-carbinolamine as depicted in Scheme 1 is tenable.

\section{References}

1. Lazarus R.A. Benkovic S.J. Kaufman S. Phenylalanine Hydroxylase Stimulator Protein Is a $4 \mathrm{a}$-Carbinolamine Dehydratase. J. Biol. Chem. 1983; 258: 10960-10962.

2. Mendel D.B. Khavari P.A. Conley P.B. et al. Characterization of a Cofactor That Regulates Dimerization of a Mammalian Homeodomain Protein. Science 1991; 254: 1762-1767.

3. Köster S. Thöny B. Macheroux P. et al. Human pterin-4a-carbinolamine dehydratase/dimerization cofactor of hepatocyte nuclear factor- $1 \alpha$ : Characterization and kinetic analysis of wild-type and mutant enzymes. Eur. J. Biochem. 1995; 231: 414-423.

4. Köster S. Thöny B. Macheroux P. Curtius H.-Ch. Heizmann C.W. Ghisla S. Characterization of $\mathrm{Hu}-$ man Pterin-4a-carbinolamine Dehydratase/Dimerization Cofactor of Hepatocyte Nuclear Factor- $1 \alpha$, and of the Cys81-mutant Involved in Hyperphenylalaninæmia. Pteridines 1995; 6: 123-125.

5. Ficner R. Sauer U.H. Stier G. Suck D. Three-dimensional structure of the bifunctional protein PCD/ $\mathrm{DCoH}$, a cytoplasmic enzyme interacting with transcription factor HNFl. EMBO Journal 1995; 14: 2034-2042.

6. Endrizzi J.A. Cronk J.D. Wang W. Crabtree G.R. Alber T. Crystal Structure of DCoH, a Bifunctional, Protein-Binding Transcriptional Coactivator. Science 1995; 268: 556-559.

7. Köster S. Stier G. Ficner R. et al. Location of the active site and proposed catalytic mechanism of pterin4a-carbinolamine dehydratase. Eur. J. Biochem.: submitted.

8. Bailey S.W. Chandrasekaran R.Y. Ayling J.E. Synthesis of Tetrahydropteridine C6-Stereoisomers, Including $\mathrm{N}^{5}$-Formyl-(6S)-tetrahydrofolic Acid. J. Org. Chem. 1992; 57: 4470-4477.

9. Sayer J.M. Peskin M. Jencks W.P. Imine-Forming Elimination Reactions. I. General Base and Acid Catalysis and Influence of the Nitrogen Substituent on Rates and Equilibria for Carbinolamine Dehydration. J. Am. Chem. Soc., 1973; 95: 4277-4287.

10. Evans S.V. SETOR: hardware lighted three-dimensional solid model representation of macromolecules. J. Mol. Graphics 1993; 11: 134-138. 